\title{
The effect of probiotics, prebiotics or synbiotics on metabolic outcomes in individuals with diabetes: a systematic review and meta-analysis
}

\author{
Patricia M. Bock ${ }^{1,2,3}$ (D) Gabriela H. Telo ${ }^{4}$ (D) Rafaela Ramalho $^{5}$ (D) Mariana Sbaraini $^{1}$ (D) $\cdot$ Gabriel Leivas $^{1}$ (D) \\ Andreza F. Martins ${ }^{5}$ (D) Beatriz D. Schaan ${ }^{1,3,6}$
}

Received: 27 May 2020 / Accepted: 12 August 2020 / Published online: 13 October 2020

(C) Springer-Verlag GmbH Germany, part of Springer Nature 2020

\begin{abstract}
Aims/hypothesis The aim was to conduct a systematic review and meta-analysis of randomised controlled clinical trials assessing the effect of probiotic, prebiotic or synbiotic supplementation on gut microbiota and glucose control and lipid levels in individuals with diabetes.

Methods MEDLINE, EMBASE and the Cochrane Library were searched. The eligibility criteria for the studies was involvement of participants with a diagnosis of type 1 or type 2 diabetes. Metabolic outcomes (glucose control, insulinaemia, and lipid profile) of any probiotic, prebiotic or synbiotic supplementation related to modification of gut microbiota (prebiotics, probiotics and synbiotics) were analysed. We provided a narrative synthesis and meta-analysis of the findings on metabolic outcomes from the studies. Metabolic outcomes were extracted post-intervention and expressed as mean differences (MDs) and 95\% CIs between treatment and comparator groups. We pooled the results using a random-effects meta-analysis. The meta-analysis was conducted using Review Manager (RevMan) software.

Results After the removal of duplicates and ineligible studies, 5219 studies were retained for review of titles and abstracts. The number of articles was reduced to 130 by review, for which the full-text articles were obtained and reassessed, 38 of which were included in the final meta-analysis. Overall, the use of prebiotics, probiotics or synbiotics reduced $\mathrm{HbA}_{1 \mathrm{c}}$ levels, but did not reach the threshold for significance $(-2.17 \mathrm{mmol} / \mathrm{mol}, 95 \% \mathrm{CI}-4.37,0.03 ; p=0.05$, $\left[-0.20 \%, 95 \% \mathrm{CI}-0.40\right.$ to $\left.\left.0.00 ; p=0.05, I^{2}=66 \%\right]\right)$ and had no effect on LDL-cholesterol levels $(-0.05 \mathrm{mmol} / \mathrm{l} ; 95 \%$ CI $\left.-0.14,0.05, p=0.35, I^{2}=37 \%\right)$. However, their consumption decreased levels of fasting blood glucose $(-0.58 \mathrm{mmol} / \mathrm{l}$; $95 \%$ CI $\left.-0.86,-0.30 ; p<0.01, I^{2}=60 \%\right)$, total cholesterol $\left(-0.14 \mathrm{mmol} / \mathrm{l} ; 95 \% \mathrm{CI}-0.26,-0.02, p=0.02, I^{2}=39 \%\right)$, triacylglycerols $\left(-0.11 \mathrm{mmol} / \mathrm{l} ; 95 \% \mathrm{CI}-0.20,-0.02, p=0.01, I^{2}=21 \%\right)$ and insulinaemia $(-10.51 \mathrm{pmol} / 1 ; 95 \% \mathrm{CI}$ $\left.-16.68,-4.33, p<0.01, I^{2}=74 \%\right)$, and increased HDL-cholesterol levels $\left(0.04 \mathrm{mmol} / \mathrm{l} ; 95 \% \mathrm{CI} 0.01,0.07, p<0.01, I^{2}=24 \%\right)$.
\end{abstract}

Electronic supplementary material The online version of this article (https://doi.org/10.1007/s00125-020-05295-1) contains peer-reviewed but unedited supplementary material, which is available to authorised users.

Patricia M. Bock

patriciabock74@gmail.com; pbock@hcpa.edu.br

1 Department of Internal Medicine, Faculty of Medicine, Graduate Program in Medical Sciences: Endocrinology, Universidade Federal do Rio Grande do Sul, Porto Alegre, Brazil

2 Faculdades Integradas de Taquara, Taquara, Brazil

3 National Institute of Science and Technology for Health Technology Assessment (IATS) - CNPq/Brazil, Hospital de Clínicas de Porto Alegre, Porto Alegre, Brazil
4 Department of Internal Medicine, Faculty of Medicine, Pontifícia Universidade Católica do Rio Grande do Sul, Porto Alegre, Brazil

5 Department of Microbiology, Immunology and Parasitology, Health Basic Science Institute, Universidade Federal do Rio Grande do Sul, Porto Alegre, Brazil

6 Endocrine Division, Hospital de Clínicas de Porto Alegre, Porto Alegre, Brazil 


\section{Research in context}

\section{What is already known about this subject?}

- Gut microbiota may play a role in the development of obesity and diabetes mellitus; bioactive agents, such as probiotics, prebiotics or synbiotics, could play a role in the prevention and treatment of diabetes

What is the key question?

- Is probiotic, prebiotic or synbiotic supplementation associated with improved glucose control and lipid levels in individuals with diabetes?

What are the new findings?

- Consumption of prebiotics, probiotics or synbiotics did not lead to changes in $\mathrm{HbA}_{1 c}$ or LDL-cholesterol levels

- Consumption of prebiotics, probiotics and synbiotics decreased fasting blood glucose, total cholesterol, triacylglycerol levels and insulinaemia

- Consumption of prebiotics, probiotics and synbiotics increased HDL-cholesterol levels

How might this impact on clinical practice in the foreseeable future?

- Consumption of probiotics, prebiotics and synbiotics may be a potential adjuvant treatment for improving metabolic outcomes

Conclusions/interpretation In individuals with diabetes mellitus, supplementation with probiotics, prebiotics or synbiotics improved metabolic variables, although the magnitude of this effect is low. Our results suggest that consumption of probiotics, prebiotics or synbiotics may be a potential adjuvant treatment for improving metabolic outcomes.

Registration PROSPERO ID CRD42017080071.

Keywords Blood glucose $\cdot$ Cholesterol $\cdot$ Diabetes mellitus $\cdot \mathrm{HbA}_{1 \mathrm{c}} \cdot$ Lactobacillus $\cdot$ Meta-analysis $\cdot$ Microbiota $\cdot$ Systematic review $\cdot$ Triacylglycerols

\section{Abbreviations \\ LPS Lipopolysaccharide \\ MD Mean difference \\ RoB2 Risk-of-bias tool}

\section{Introduction}

Diabetes mellitus has become a global pandemic, largely because of the increasing prevalence of obesity and unhealthy lifestyles [1]. Recent studies suggest that the gut microbiota may play a role in obesity [2], metabolic syndrome and diabetes mellitus [3]. Altered microbiota composition features in the progression of type 2 diabetes, with an increasing loss of gut microbial diversity, which is related to insulin resistance and enhanced circulating inflammation markers [4]. Although controversial, because it has not been demonstrated in humans, altered microbiota would be related to increased intestinal permeability, development of metabolic endotoxaemia and inflammation, presumably because of the translocation of bacterial products, such as lipopolysaccharides (LPS) originating in the gut, which in turn would trigger the development of diabetes [5]. In women, type 2 diabetes is positively associated with metabolic endotoxaemia, and IL-6 levels are found to be increased [6]. Thus, the gut microbiota is suggested to drive the pathogenesis of metabolic diseases, including type 2 diabetes.

Bioactive agents, such as probiotics (live microorganisms that when administered in adequate amounts may confer a health benefit on the host) [7], prebiotics (a substrate that is selectively utilised by the microorganisms of the host, conferring a health benefit) [8] or synbiotics (a probiotic-prebiotic combination), could improve the gut microbiota. This change in gut microbiota could, at least to some extent, improve the metabolic control of individuals with type 2 diabetes [9], reducing plasma levels of bacterially derived LPS and improving the gut barrier function, as shown in genetically obese mice [10]. Thus, these bioactive agents could playing a role in the prevention and treatment of diabetes.

Several experimental studies on animal models of diabetes (fructose-induced, alloxan-induced, high-fat diet-induced, genetic models) have demonstrated the benefits of specific probiotic bacterial strains on glucose control. Benefits have been shown with probiotics containing Lactobacillus 
acidophilus and Lactobacillus casei [11], Lactobacillus plantarum TN627 strain [12], Lactobacillus plantarum DSM 15313 [13], Lactobacillus gasseri BNR17 [14], Lactobacillus reuteri [15] and Lactobacillus rhamnosus, but not with Lactobacillus bulgaricus [16]. Other metabolic effects have been reported with the use of probiotics in experimental studies on diabetes. Bifidobacterium lactis was associated with low levels of lipids and insulinaemia [17]; L. casei CCFM0412 improved glucose tolerance, lowered lipid levels, enhanced immune regulation and reduced oxidative stress [18]; and Lactobacillus johnsonii led to upregulated expression of proteins involved in intercellular tight junction assembly and maintenance in the gut [19].

Studies on consumption of probiotics or synbiotics by individuals with diabetes have provided conflicting results, with some reporting improved metabolic control [20-22] and others not $[23,24]$. A recent systematic review suggested that supplementation with probiotics and synbiotics could be beneficial in lowering fasting blood glucose in adults with high baseline fasting blood glucose [25]; however, the included studies evaluated individuals with other conditions in addition to those with diabetes, and no information was provided on glucose control and lipid profile. Because of the lack of consistent data in the literature, the current systematic review and meta-analysis aimed to evaluate the impact of probiotic, prebiotic or synbiotic supplementation related to modification of gut microbiota on glucose control and lipid levels in individuals with diabetes. This study may have important implications for the development of a probiotic treatment for diabetes and may form a rational basis for the selection of specific probiotic agents to boost gut mucosal regulatory responses.

\section{Methods}

The Preferred Reporting Items for Systematic Reviews and Meta-Analysis (PRISMA) Statement was followed as a guideline for conducting and reporting this systematic review and meta-analysis [26].

This systematic review is registered in the International Prospective Register for Systematic Review (PROSPERO) database under number CRD42017080071.

Eligibility criteria The inclusion criteria were as follows: studies with adult participants with type 1 or 2 diabetes diagnosis and a focus on metabolic outcomes (glucose control, insulinaemia and lipid profile) that involved any probiotic, prebiotic or synbiotic supplementation or combination of interventions with the aim of adjusting the gut microbiota. Studies reporting gestational or other diabetes types were excluded. Because of the diverse range of possible interventions, to avoid exclusion of relevant data we did not restrict the intervention type in the search, but only randomised clinical trials were included. Only studies published in English, Spanish or Portuguese were included. We did not include conference abstracts.

Information sources and search In the article search process, we used the terms 'diabetes mellitus' and 'microbiota' in the selected databases. To extend our search strategy, we did not use any terms referring to the control or study design. MEDLINE, EMBASE and the Cochrane Library were searched using a combination of MeSH headings, keywords and related entry terms to identify the potentially relevant studies. The complete search strategy used for the PubMed database is shown in electronic supplementary material (ESM) text 1.

The search process was completed by October 2017, and updated in September 2019 and, again, in July 2020. After combining the search results of different databases, the duplicates were removed. Records were managed using EndNote X7. Manual search (i.e., reference lists and citation searching) of studies fulfilling the eligibility criteria was also carried out.

Study selection and data collection process Two authors (PMB and RR) independently screened the titles and abstracts of all the studies generated by the search to identify studies that met the inclusion criteria outlined above. The reviewers were not blinded to the authors, institutions or the name of the journals the manuscripts were published in. Papers with abstracts that did not provide enough information regarding the inclusion and exclusion criteria were retrieved for full-text evaluation. The full-text articles were assessed independently by the same two authors (PMB and RR) to decide whether or not they should be retained. Any disagreement was resolved by a third independent author (GHT).

A standardised, pre-piloted form (Excel) was used to extract data from the included studies for evidence synthesis. The following information was extracted from included studies: first author's name, publication year, title, objective, intervention type, study design, daily dose, pharmaceutical formulation, sample size, follow-up time, disease duration and evaluated outcomes. Means \pm standard deviations post-intervention were extracted for continuous variables related to metabolic evaluation (levels of plasma glucose and $\mathrm{HbA}_{1 \mathrm{c}}$, insulinaemia, lipid profile) and BMI. Relevant data were extracted from studies by two separate investigators (PMB and RR). Any disagreement was resolved by a third independent author (AFM). The corresponding author was contacted as needed to obtain data not included in the published report.

Risk of bias and publication bias assessment The risk-of-bias assessment in the included studies was performed according to the revised Cochrane risk-of-bias tool (RoB2) [27]. A standardised, pre-piloted form (Excel) was used to extract data from the included studies for assessment of study quality. Each study was evaluated for the following items: bias arising 


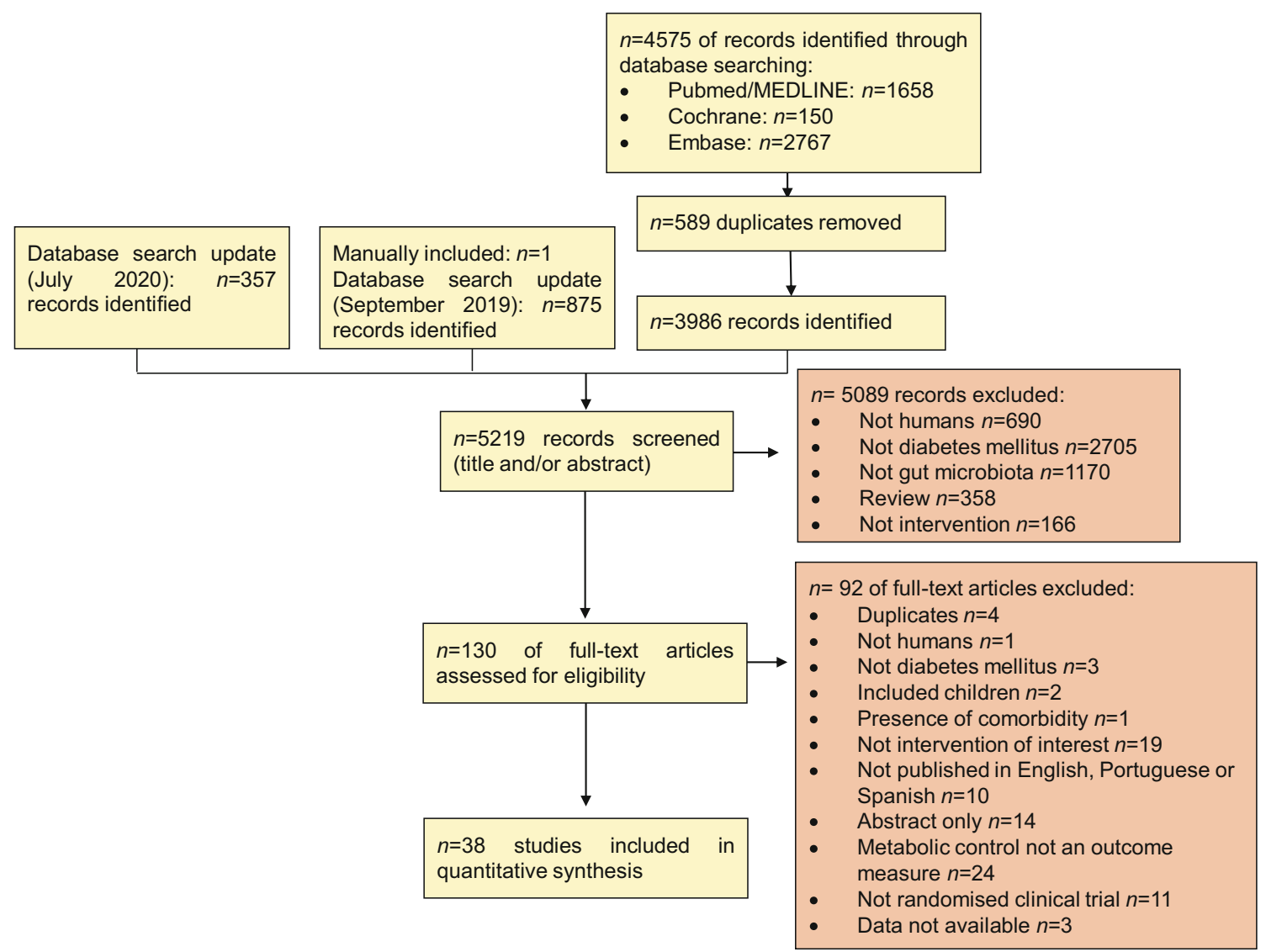

Fig. 1 Flowchart to illustrate how articles were identified and selected for inclusion in the meta-analysis

from the randomisation process, bias because of deviations from intended interventions (effect of assignment to intervention), bias because of missing outcome data, bias in measurement of the outcome, and bias in selection of the reported result. Each domain was composed of multiple questions, and using an algorithm, they were judged as having low risk, some concerns or high risk of bias. The risk-of-bias assessment was performed by two independent reviewers (MS and GL). Publication bias was assessed using a contour-enhanced funnel plot of each trial's effect size against the standard error of the estimate.

Data analysis We aimed to provide a narrative synthesis of the findings from the included studies, structured around the type of outcome. The meta-analysis was conducted using Review Manager (RevMan) software, version 5.3 (The Nordic Cochrane Centre, The Cochrane Collaboration, Copenhagen, Denmark). Metabolic outcomes and BMI were expressed as mean differences (MDs) and 95\% CIs between treatment and comparator groups. We pooled the results using a randomeffects model.

Statistical heterogeneity of the treatment effect among studies was assessed using both the $\chi^{2}$ test and the $I^{2}$ statistic. We considered an $I^{2}$ value of $>75 \%$ indicative of considerable heterogeneity, according to the Cochrane Handbook for
Systematic Reviews of Interventions [28]. A $p$ value of $<0.05$ was considered to indicate a statistically significant effect. We explored heterogeneity between studies using two strategies. First, we re-ran the meta-analyses by assessing the effect of individual studies on the overall results of the metaanalysis, removing one study at a time to check if any specific study explained the heterogeneity. Second, we performed sensitivity analyses to evaluate subgroups of studies most likely to yield valid estimates of the intervention based on prespecified relevant information as follows: (1) specific probiotic species, excluding trials that did not involve Lactobacillus; (2) risk of bias, excluding high-risk trials; (3) presence vs absence of a simultaneous cointervention; (4) placebo use; and (5) blinding.

\section{Results}

Study selection The electronic search returned 4575 potentially relevant studies from searches of the databases (PubMed/ MEDLINE $=1658$, Cochrane $=150$, and EMBASE $=2767$ ). Additional searches identified 1232 studies (last search was conducted in July 2020). One additional study was identified by manual search of the reference lists of the selected studies and was included. After the removal of duplicates and 


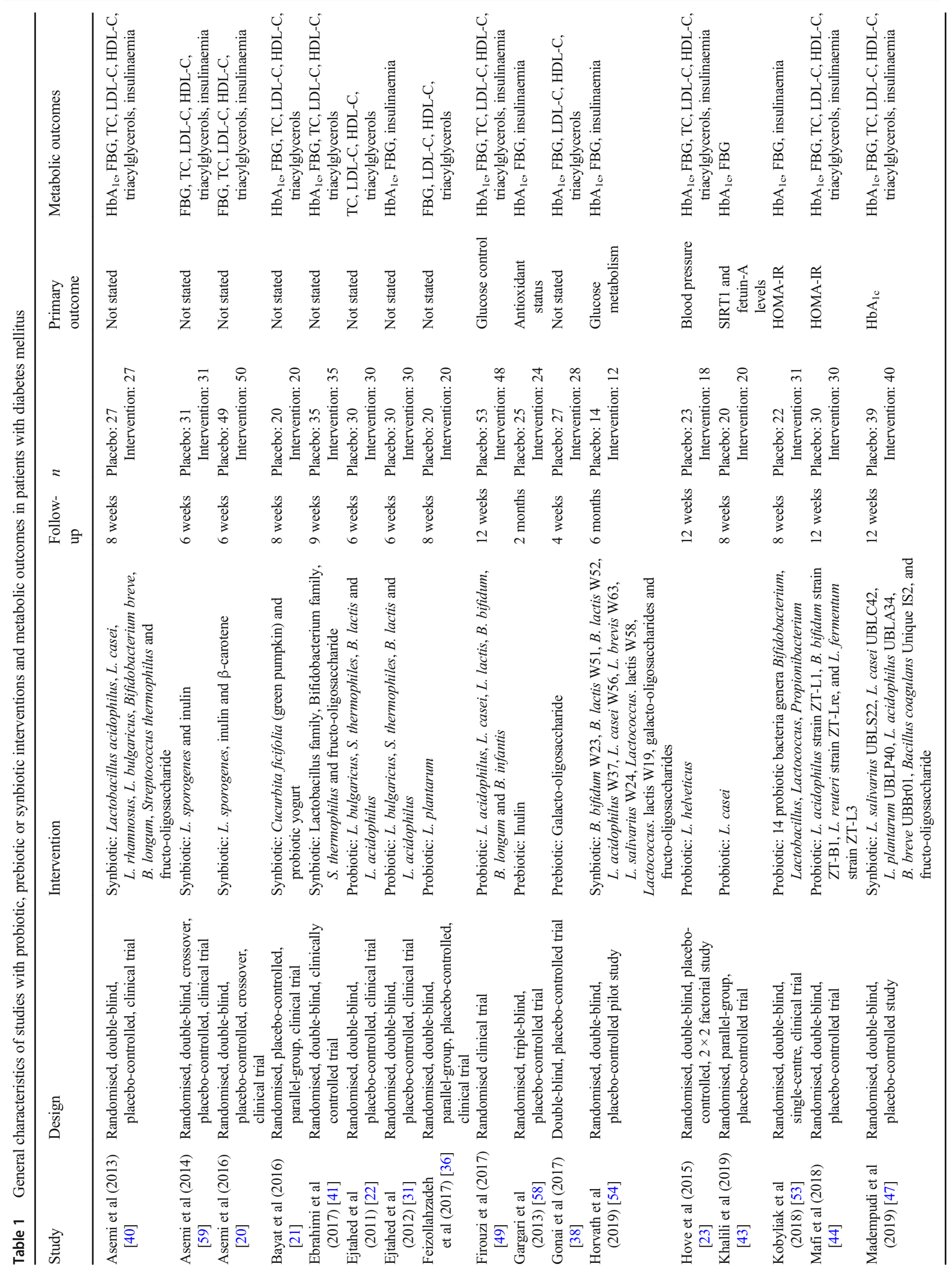




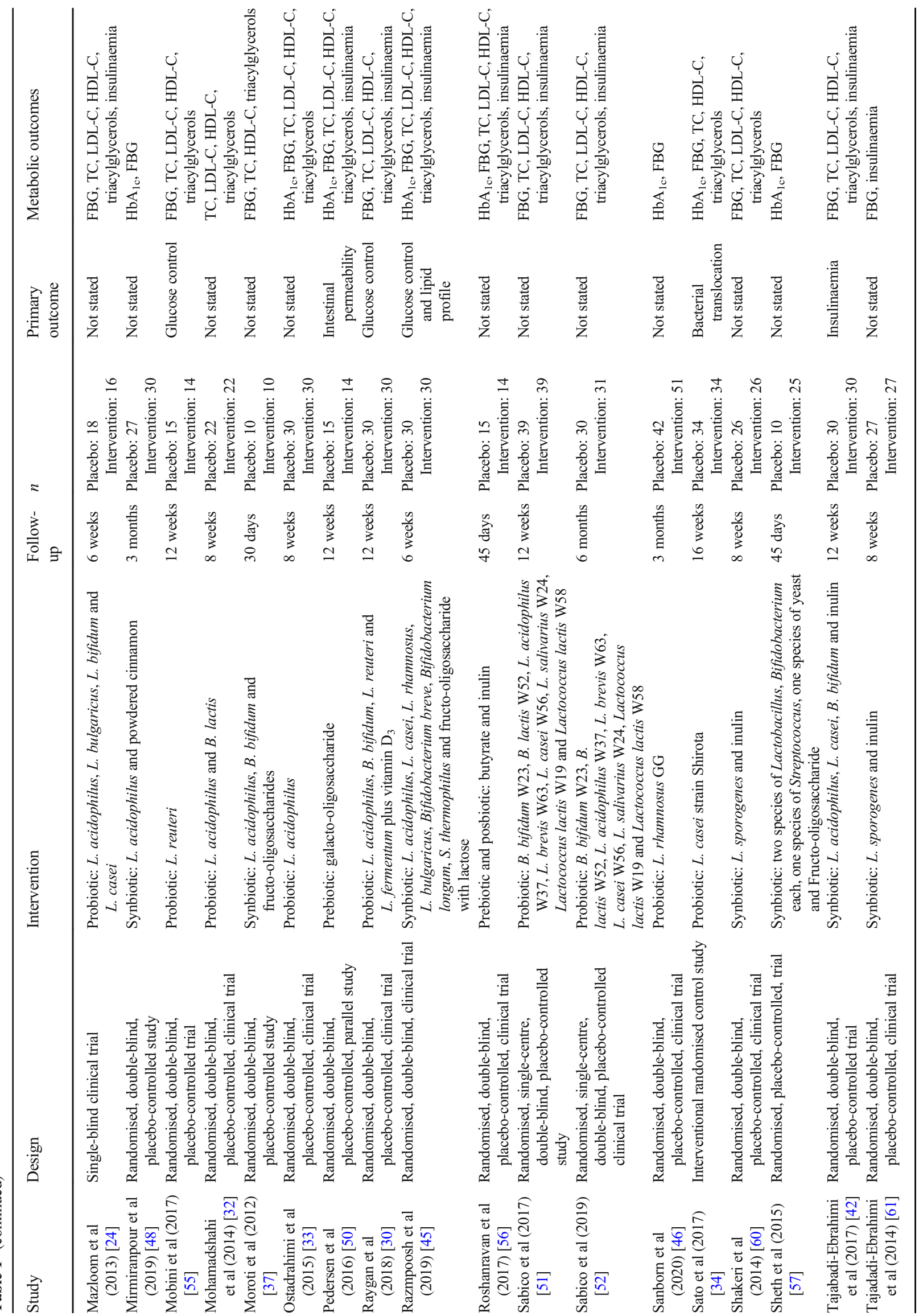




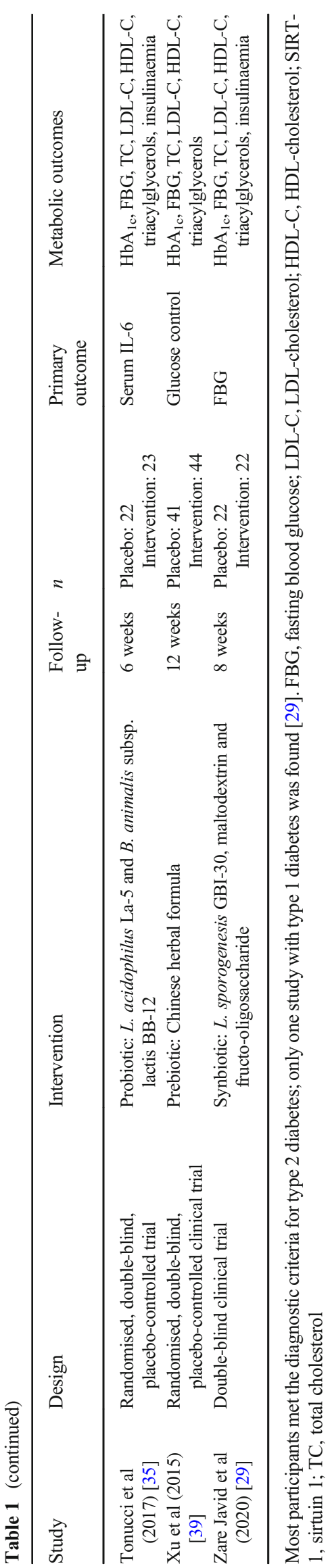


ineligible studies, 5219 studies were retained for review of titles and abstracts. The number of articles was reduced to 130 by review, for which the full-text articles were obtained and reassessed, 38 of which were included in the final metaanalysis. A detailed flowchart showing the study search and selection process is presented in Fig. 1.

Study characteristics The characteristics of the included studies are described in Table 1. A total of 2086 randomised participants from the eligible trials were included in this meta-analysis. Most participants met the diagnostic criteria for type 2 diabetes as set out by each study; only one study included individuals with type 1 diabetes [29]. The first trial to evaluate the impact of a probiotic intervention on metabolic control was published in 2011, evaluating lipid levels as outcomes. Twenty-eight of the included studies were published in the last 5 years.

The durations of the interventions varied from 30 days to 6 months. In 18, 5 and 15 trials, probiotics, prebiotics and synbiotics were used as the intervention. In 12 trials, supplementation involved a single probiotic species, while 20 studies used multiple strains of probiotic bacteria. No study in this meta-analysis included more than one dose of probiotics. Only two studies presented data on co-interventions; one used esomeprazole [23], the other, vitamin $\mathrm{D}_{3}$ [30]. No major adverse effects were reported (ESM Table 1).

Concerning liquid formulations, five trials used probiotic yogurt [21-23, 31, 32], three studies used fermented milk [33-35], one used soy milk [36], one used a shake [37], one used a syrup [38] and one used a decoction [39] as the carrier. Regarding solid pharmaceutical formulations, ten studies used capsules [24, 40-48], six studies used sachets [49-54], and five used powder package [29, 55-58] as the source of probiotics. Four studies used other types of foods for supplementation [20, 59-61]. One study did not report the formulation used [30].

In all the studies, a final assessment was carried out and the following outcomes were reported: $\mathrm{HbA}_{1 \mathrm{c}}(n=13$ for more than 12 weeks of treatment; one that evaluated individuals with type 1 diabetes was not included in the insulinaemia analysis), fasting blood glucose $(n=36)$, insulinaemia $(n=$ $22)$, total cholesterol $(n=27)$, LDL-cholesterol $(n=27)$, HDL-cholesterol $(n=29)$ and triacylglycerols $(n=29)$.

Risk of bias and publication bias assessment All included studies were assessed for methodological quality using the Cochrane RoB2 tool (ESM Fig. 1, ESM Table 2).

The risk of bias as per the RoB2 evaluation tool was overall low in $13.2 \%$ of studies, indicated some concerns in $47.4 \%$, and high in $39.5 \%$ of the studies. Most of the studies had a low risk of bias because of deviations from intended interventions (92\%), missing outcome data (82\%) and measurement of the outcomes $(87 \%)$. In the domain of bias arising from the
Fig. 2 Absolute changes in (a) $\mathrm{HbA}_{1 \mathrm{c}}$, (b) fasting blood glucose and (c) insulinaemia in individual studies on supplementation with probiotics, prebiotics or synbiotics. 'IV, Random' refers to a random-effects metaanalysis with weights based on inverse variances

randomisation process, $50 \%$ of the studies were considered as indicating some concerns. In selection of the reported result, $48 \%$ of the studies were judged as having low risk of bias, mostly because of an incomplete or absent study protocol.

The possibility of publication bias was evaluated by using a funnel plot for the primary outcome, $\mathrm{HbA}_{1 \mathrm{c}}$ and fasting blood glucose (ESM Fig. 2a, b). The points for the missing studies would be on the bottom left side of the plot. Since most of this area contains regions of high significance, publication bias is unlikely to be the underlying cause of this asymmetry. Given the limited number of studies included in the primary outcome meta-analysis, no further tests were run to distinguish between chance and real asymmetry.

Synthesis of results The data from the meta-analysis on the impact of probiotics and synbiotics on glucose control are presented in Fig. 2 and on lipid profile in Fig. 3. Only studies with a duration of more than 12 weeks were considered for the meta-analysis of $\mathrm{HbA}_{1 \mathrm{c}}$; probiotics/prebiotics/synbiotics did not decrease $\mathrm{HbA}_{1 \mathrm{c}}$ levels $(-2.17 \mathrm{mmol} / \mathrm{mol}, 95 \% \mathrm{CI}-4.37$, $0.03 ; p=0.05 ; p$ for heterogeneity $<0.01[-0.20 \%, 95 \% \mathrm{CI}$ -0.40 to $0.00 ; p=0.05, I^{2}=66 \%$ ], Fig. $2 \mathrm{a}$ ).

Consumption of probiotics, prebiotics or synbiotics decreased fasting blood glucose levels $(-0.58 \mathrm{mmol} / 1,95 \%$ CI $-0.86,-0.30 ; p<0.01, I^{2}=60 \% ; p$ for heterogeneity $<0.01$, Fig. 2b) and insulinaemia $(-10.51 \mathrm{pmol} / 1 ; 95 \% \mathrm{CI}$ $-16.68,-4.33, p<0.01, I^{2}=74 \%$; $p$ for heterogeneity $<0.01$, Fig. 2c). The study that evaluated individuals with type 1 diabetes was not included in the insulinaemia analysis. Probiotics, prebiotics or synbiotics had no effect on BMI $\left(-0.06 \mathrm{~kg} / \mathrm{m}^{2}, 95 \% \mathrm{CI}-0.53,0.41 ; p=0.81, I^{2}=0 \% ; p\right.$ for heterogeneity $=0.87)($ ESM Fig. 3$)$.

Consumption of probiotics, prebiotics or synbiotics decreased total cholesterol $(-0.14 \mathrm{mmol} / \mathrm{l} ; 95 \% \mathrm{CI}-0.26$, $-0.02, p=0.02, I^{2}=39 \% ; p$ for heterogeneity $=0.02$; Fig. $3 \mathrm{a})$ and triacylglycerol levels $(-0.11 \mathrm{mmol} / \mathrm{l} ; 95 \% \mathrm{CI}-0.20$, $-0.02, p=0.01, I^{2}=21 \%$; $p$ for heterogeneity $=0.16$; Fig. $3 \mathrm{~d}$ ), while HDL-cholesterol was increased $(0.04 \mathrm{mmol} / \mathrm{l} ; 95 \% \mathrm{CI}$ $0.01,0.07, p<0.01, I^{2}=24 \%$; $p$ for heterogeneity $=0.12$; Fig. $3 \mathrm{c})$. However, consumption of probiotics, prebiotics or synbiotics had no effect on LDL-cholesterol levels $\left(-0.05 \mathrm{mmol} / \mathrm{l} ; 95 \% \mathrm{CI}-0.14,0.05, p=0.35, I^{2}=37 \% ; p\right.$ for heterogeneity $=0.03$; Fig. 3b).

When studies were omitted individually from the metaanalysis to assess possible individual influences on outcomes, the heterogeneity was unchanged. The sensitivity analyses conducted to assess results using Lactobacillus, presence vs 
a

Study

Firouzi et al (2017) [49] Gargari et al (2013) [58] Hove et al (2015) [23] Madempudi et al (2019) [47] Mafi et al (2018) [44] Mirmiranpour et al (2019) [48] Mobini et al (2017) [55] Sanborn et al (2020) [46] Sato et al (2017) [34] Xu et al (2015) [39]

\begin{tabular}{lrrrrr}
\multicolumn{2}{c}{ Experimental } & \multicolumn{4}{c}{ Control } \\
Mean & SD & $n$ & Mean & SD & $n$ \\
56.50 & 15.3 & 48 & 56.40 & 18.6 & 53 \\
60.60 & 7.5 & 24 & 67.20 & 11.9 & 25 \\
67.20 & 47.0 & 12 & 63.90 & 24.0 & 14 \\
54.10 & 6.6 & 18 & 60.60 & 9.8 & 23 \\
60.60 & 8.6 & 40 & 67.20 & 14.8 & 39 \\
50.80 & 4.4 & 30 & 51.90 & 3.3 & 30 \\
60.20 & 12.1 & 30 & 69.20 & 6.4 & 27 \\
66.10 & 10.9 & 14 & 60.60 & 6.6 & 15 \\
53.00 & 12.2 & 14 & 48.60 & 7.9 & 15 \\
37.90 & 3.7 & 51 & 39.30 & 6.1 & 42 \\
54.10 & 7.7 & 34 & 51.90 & 5.5 & 34 \\
56.90 & 8.6 & 44 & 60.50 & 12.7 & 41
\end{tabular}

Random effects model 359

Heterogeneity: $l^{2}=66 \%, \tau^{2}=8.1879, \chi_{11}^{2}=32.35(p<0.01)$ 358

Test for overall effect: $z=-1.93(p=0.05)$ Horvath et al (2019) [54] Pedersen et al (2016) [50]

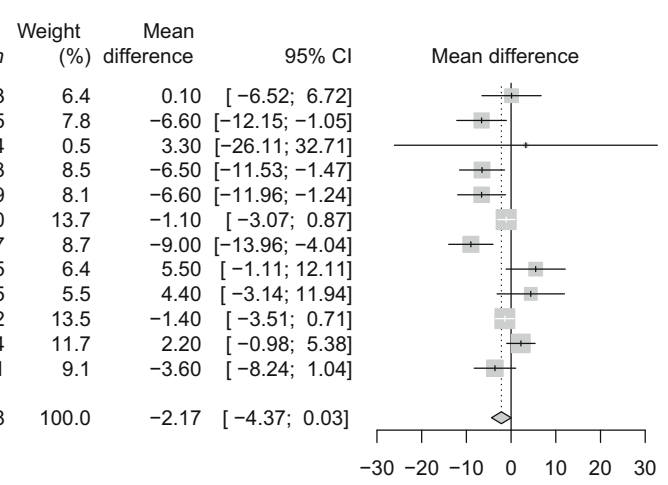

b Study

Asemi et al (2013) [40] Asemi et al (2014) [59] Asemi et al (2016) [20] Bayat et al (2016) [21] Ebrahimi et al (2017) [41]
Ejtahed et al (2012) [31] Feizollahzadeh et al (2017) [36] Firouzi et al (2017) [49] Gargari et al (2013) [58] Gonai et al (2017) [38] Horvath et al (2019) [54 Hove et al (2015) [23] Khalili et al (2019) [43] Kobyliak et al (2018) [53] Madempudi et al (2019) [47] Mafi et al (2018) [44] Mazloom et al (2013) [24] Mirmiranpour et al (2019) [48] Mobini et al (2017) [55] Moroti et al (2012) [37] Ostadrahimi et al (2015) [33] Pedersen et al (2016) [50] Raygan et al (2018) [30] Razmpoosh et al (2019) [45] Roshanravan et al (2017) [56] Sabico et al (2017) [51]
Sabico et al (2019) [52] Sabico et al (2019) [52] Sanborn et al (2020) [46] Sato et al (2017) [34] Shakeri et al (2014) [60] Sheth et al (2015) [57] Tajabadi-Ebrahimi et al (2017) [42] Tajadadi-Ebrahimi et al (2014) [61] Tonucci et al (2017) [35] $\mathrm{Xu}$ et al (2015) [39] Zare Javid et al (2020) [29]

\begin{tabular}{rr} 
Experimental \\
Mean & SD \\
8.07 & 2.7 \\
9.45 & 3.9 \\
7.54 & 2.1 \\
6.38 & 1.2 \\
7.33 & 2.1 \\
7.35 & 2.4 \\
7.33 & 3.9 \\
7.21 & 1.2 \\
8.14 & 1.1 \\
7.68 & 1.7 \\
10.43 & 4.6 \\
7.91 & 1.9 \\
7.54 & 2.4 \\
8.31 & 2.5 \\
7.01 & 1.5 \\
6.85 & 1.7 \\
8.81 & 3.6 \\
8.21 & 0.2 \\
12.29 & 2.7 \\
6.48 & 1.0 \\
7.73 & 2.6 \\
6.79 & 1.5 \\
6.38 & 2.6 \\
7.31 & 1.7 \\
8.82 & 2.4 \\
8.49 & 3.5 \\
7.19 & 2.8 \\
5.36 & 0.6 \\
7.39 & 1.2 \\
7.05 & 2.2 \\
8.15 & 0.9 \\
7.21 & 2.2 \\
7.31 & 2.0 \\
8.41 & 2.4 \\
7.55 & 1.3 \\
9.08 & 4.2 \\
\hline 61$]$
\end{tabular}

Control $n$ eight Mean
$95 \% \mathrm{Cl}$ $-0.99 \quad[-2.65 ; 0.67]$ $-0.13 \quad[-2.00 ; 1.74]$ $-0.59 \quad[-1.63 ; 0.45]$ $-2.81 \quad[-3.95 ;-1.67]$ $0.10 \quad[-0.79 ; 0.99]$ $-0.17 \quad[-1.15 ; 0.81]$ $-0.16 \quad[-2.70 ; 2.38]$ $-1.00[-1.71 ;-0.29]$ $\begin{array}{ll}-1.00 & {[-1.71 ;-0.29]} \\ -0.52 & {[-1.06 ; 0.02]}\end{array}$ $0.01 \quad[-0.94 ; 0.96]$ $1.38 \quad[-1.64 ; 4.40]$ $-2.00 \quad[-3.65 ;-0.35]$ $-0.82 \quad[-2.20 ; 0.56]$ $-0.61 \quad[-1.90 ; 0.68]$ $-1.20[-2.21 ;-0.19]$ $-0.51 \quad[-1.36 ; 0.34]$ $-0.22 \quad[-2.74 ; 2.30]$ $-1.63[-2.12 ;-1.14]$ $0.40 \quad[-1.68 ; 2.48]$ $0.34 \quad[-0.88 ; 1.56]$ $-2.38[-4.11 ;-0.65]$ $0.30 \quad[-0.66 ; 1.26]$ $-0.53 \quad[-1.69 ; 0.63]$ $-0.80 \quad[-1.72 ; 0.12]$ $1.56 \quad[0.16 ; 2.96]$ $0.50 \quad[-1.19 ; 2.19]$ $-0.90 \quad[-2.45 ; 0.65]$ $-0.21 \quad[-0.52 ; 0.10]$ $-0.22 \quad[-0.94 ; 0.50]$ $-2.30[-4.32 ;-0.28]$ $-0.04[-0.74 ; 0.66]$ $\begin{array}{lll}-0.04 & {[-0.74 ;} & 0.66] \\ -0.81 & {[-2.46 ; 0.84]}\end{array}$ $-2.13[-3.80 ;-0.46]$ $0.87 \quad[-0.57 ; 2.31]$ $\begin{array}{ll}-1.19 & {[-1.93 ;-0.45]} \\ -0.45 & {[-2.91 ; 2.01]}\end{array}$ $-0.58[-0.86 ;-0.30]$

Random effects mode $9.08 \quad 4.2$

$-0.58$

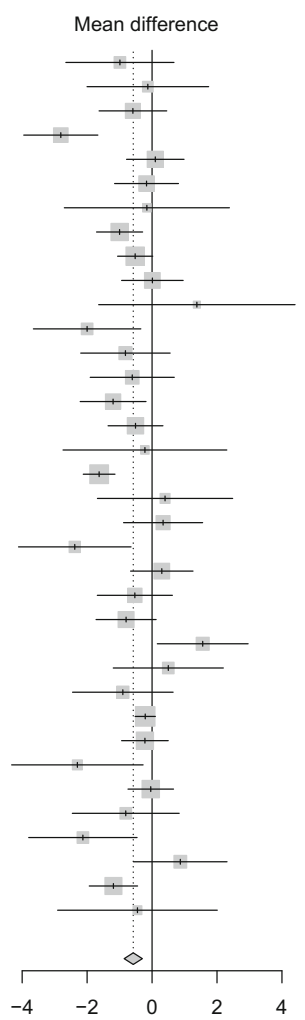

\section{C}

Study

Asemi et al (2013) [40] Asemi et al (2014) [59] Asemi et al (2016) [20] Ejtahed et al (2012) [31] Firouzi et al (2017) [49] Gargari et al (2013) [58] Horvath et al (2019) [54] Hove et al (2015) [23] Kobyliak et al (2018) [53] Madempudi et al (2019) [47] Mafi et al (2018) [44] Mazloom et al (2013) [24] Pedersen et al (2016) [50] Raygan et al (2018) [30] Razmpoosh et al (2019) [45] Roshanravan et al (2017) [56] Sabico et al (2017) [51] Sabico et al (2019) [52]

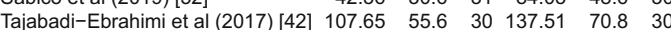
Tajadadi-Ebrahimi et al (2014) [61] $\begin{array}{lllllll}54.87 & 15.3 & 27 & 58.34 & 37.5 & 27\end{array}$ Tonucci et al (2017) [35]

\begin{tabular}{rrrrrr}
\multicolumn{4}{c}{ Experimental } & \multicolumn{4}{c}{ Control } \\
Mean & SD & $n$ & Mean & SD & $n$ \\
53.75 & 40.1 & 27 & 68.96 & 54.5 & 27 \\
48.96 & 27.9 & 31 & 62.71 & 59.0 & 31 \\
69.45 & 61.5 & 50 & 87.78 & 67.8 & 49 \\
48.41 & 31.2 & 30 & 45.14 & 24.8 & 30 \\
70.84 & 43.8 & 48 & 95.84 & 43.8 & 53 \\
64.52 & 22.2 & 24 & 93.34 & 33.3 & 25 \\
159.74 & 132.6 & 12 & 430.59 & 607.5 & 14 \\
51.95 & 38.5 & 18 & 38.96 & 30.4 & 23 \\
100.42 & 54.6 & 31 & 140.64 & 77.1 & 22 \\
53.48 & 26.1 & 40 & 62.51 & 28.8 & 39 \\
99.31 & 41.0 & 30 & 115.29 & 50.7 & 30 \\
2.43 & 3.1 & 16 & 1.74 & 0.6 & 18 \\
94.04 & 18.7 & 14 & 82.99 & 13.0 & 15 \\
75.71 & 51.4 & 30 & 92.37 & 54.9 & 30 \\
65.28 & 38.9 & 30 & 68.76 & 67.4 & 30 \\
35.42 & 11.7 & 14 & 35.98 & 7.5 & 15 \\
47.92 & 27.1 & 39 & 74.31 & 34.7 & 39 \\
42.36 & 30.6 & 31 & 84.03 & 48.6 & 30 \\
107.65 & 55.6 & 30 & 137.51 & 70.8 & 30 \\
54.87 & 15.3 & 27 & 58.34 & 37.5 & 27 \\
47.23 & 31.9 & 23 & 46.18 & 31.9 & 22
\end{tabular}

Random effects model 595
$0.01)$ Test for overall effect: $z=-3.34(p<0.01)$

599

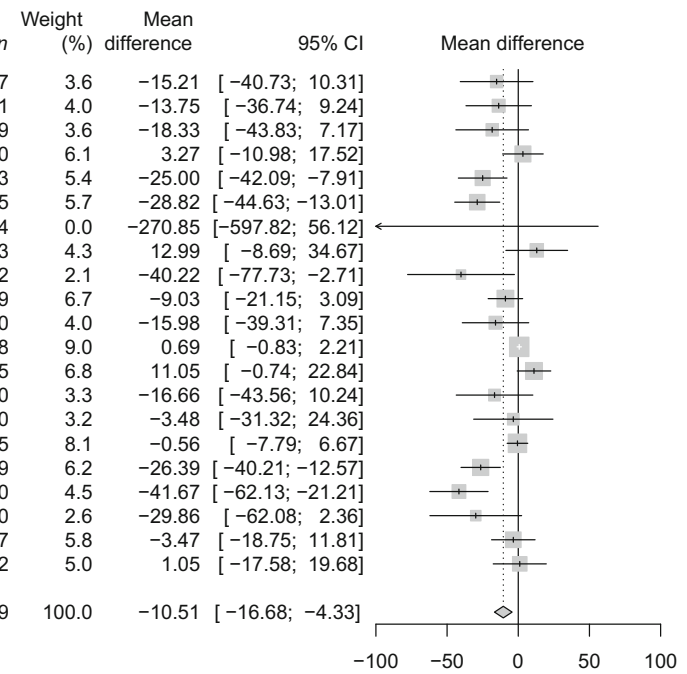


absence of a simultaneous cointervention, risk of bias, type of placebo used and blinding, slightly changed heterogeneity, with no significant overall effect on the results (data not shown).

\section{Discussion}

In the field of diabetes there is growing interest in the modulation of gut microbiota through supplementation with a

Study

Asemi et al (2013) [40]

Asemi et al (2014) [59]

Asemi et al (2016) [20]

Bayat et al (2016) [21]

Ebrahimi et al (2017) [41]

Ejtahed et al (2011) [22]

Firouzi et al (2017) [49]

Hove et al (2015) [23]

Madempudi et al (2019) [47]

Mafi et al (2018) [44]

Mazloom et al (2013) [24]

Mobini et al (2017) [55]

Mohamadshahi et al (2014) [32]

Moroti et al (2012) [37]

Ostadrahimi et al (2015) [33]

Pedersen et al (2016) [50]

Raygan et al (2018) [30]

Razmpoosh et al (2019) [45]

Roshanravan et al (2017) [56]

Sabico et al (2017) [51]

Sabico et al (2019) [52]

Sato et al (2017) [34]

Shakeri et al (2014) [60]

Tajabadi-Ebrahimi et al (2017) [42]

Tonucci et al (2017) [35]

$X u$ et al (2015) [39]

Zare Javid et al (2020) [29]

Random effects model

Heterogeneity: $I^{2}=39 \%, \tau^{2}=0.0380, \chi_{26}^{2}=42.97(p=0.02)$

Test for overall effect: $z=-2.29(p=0.02)$

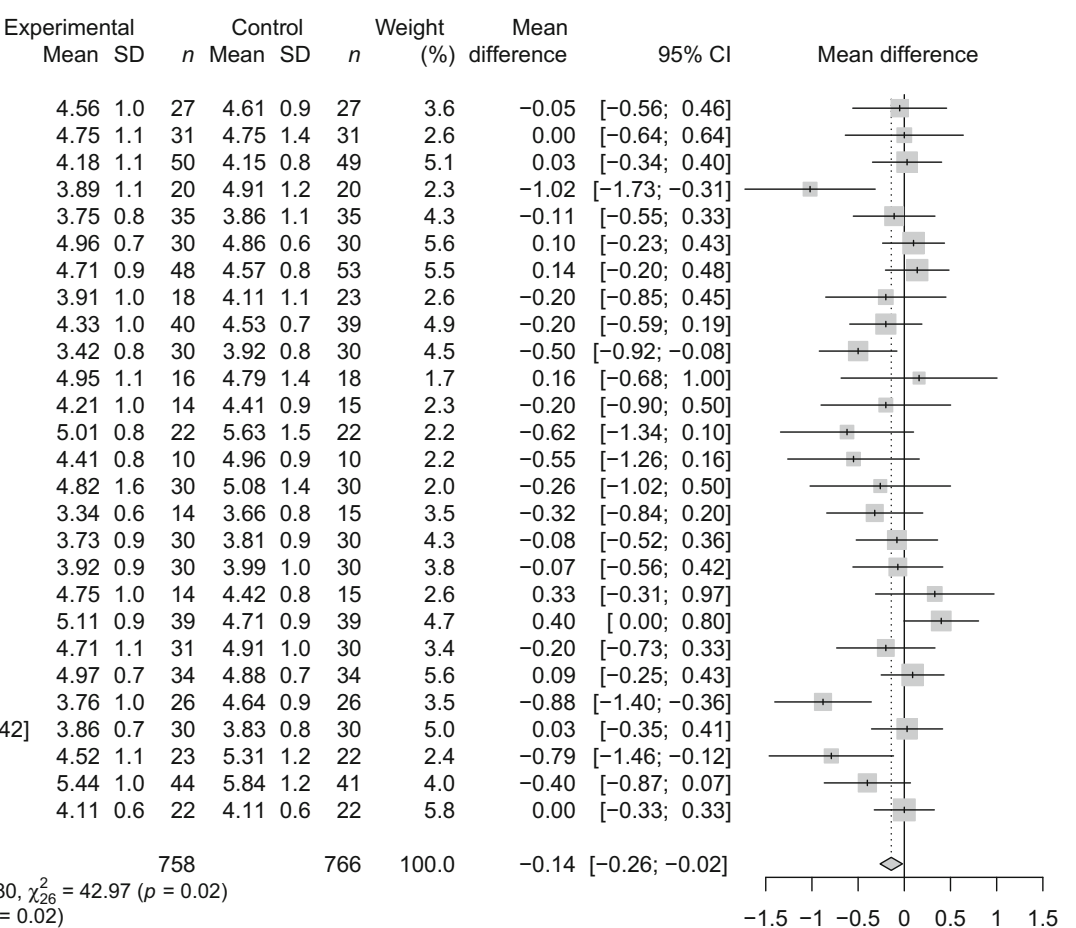

b

Study

Asemi et al (2013) [40]

Asemi et al (2014) [59]

Asemi et al (2016) [20]

Bayat et al (2016) [21]

Ebrahimi et al (2017) [41]

Ejtahed et al (2011) [22]

Feizollahzadeh et al (2017) [36]

Firouzi et al (2017) [49]

Gonai et al (2017) [38]

Hove et al (2015) [23]

Madempudi et al (2019) [47]

Mafi et al (2018) [44]

Mazloom et al (2013) [24]

Mobini et al (2017) [55]

Mohamadshahi et al (2014) [32]

Ostadrahimi et al (2015) [33]

Pedersen et al (2016) [50]

Raygan et al (2018) [30]]

Razmpoosh et al (2019) [45]

Roshanravan et al (2017) [56]

Sabico et al (2017) [51]

Sabico et al (2019) [52]

Shakeri et al (2014) [60]

Tajabadi-Ebrahimi et al (2017) [42]

Tonucci et al (2017) [35]

$\mathrm{Xu}$ et al (2015) [39]

Zare Javid et al (2020) [29]

Expe

Weight Mean

(\%) difference

$95 \% \mathrm{Cl}$

Mean difference

$-0.14[-0.58 ; 0.30]$

$0.10 \quad[-0.36 ; 0.56]$

$0.09 \quad[-0.22 ; 0.40]$

$-0.38 \quad[-0.87 ; 0.11]$

$-0.06 \quad[-0.32 ; 0.20]$

$0.05[-0.27 ; 0.37]$

$-0.57 \quad[-1.71 ; 0.57]$

$0.10 \quad[-0.20 ; 0.40]$

$0.58 \quad[0.18 ; 0.98]$

$0.10 \quad[-0.40 ; 0.60]$

$-0.01 \quad[-0.35 ; 0.33]$

$-0.38 \quad[-0.76 ; 0.00]$

$0.17 \quad[-0.36 ; 0.70]$

$0.10 \quad[-0.47 ; 0.67]$

$-0.66[-1.31 ;-0.01]$

$0.13[-0.35 ; 0.61]$

$-0.34 \quad[-0.79 ; 0.11]$

$0.00[-0.34 ; 0.34]$

$-0.07 \quad[-0.41 ; 0.27]$

$0.03 \quad[-0.51 ; 0.57]$

$0.40 \quad[0.00 ; 0.80]$

$-0.10 \quad[-0.61 ; 0.41]$

$-0.65[-1.08 ;-0.22]$

$-0.06 \quad[-0.37 ; 0.25]$

$-0.50 \quad[-1.07 ; 0.07]$

$-0.26 \quad[-0.61 ; 0.09]$

$0.07 \quad[-0.18 ; 0.32]$

$-0.05[-0.14 ; 0.05]$

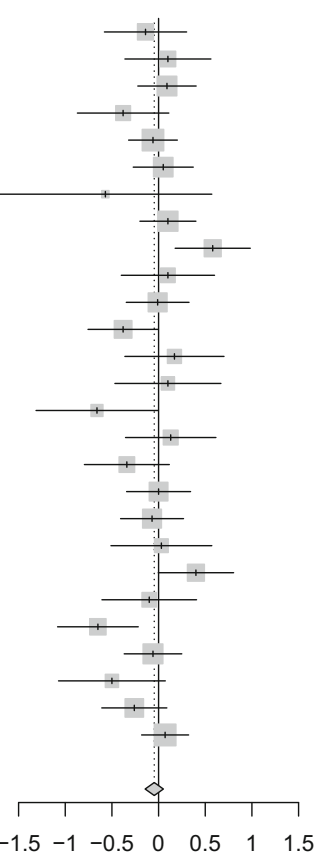

$\begin{array}{llll}\text { Random effects model } & 762 & 769 & 100.0\end{array}$

Heterogeneity: $I^{2}=37 \%, \tau^{2}=0.0234, \chi_{26}^{2}=$

$\begin{array}{lllllll}-1.5 & -1 & -0.5 & 0 & 0.5 & 1 & 1.5\end{array}$

Fig. 3 Absolute changes in lipid profile of individual studies on supplementation with probiotics, prebiotics or synbiotics. (a) Total cholesterol, (b) LDLcholesterol, (c) HDL-cholesterol (d) Triacylglycerols. 'IV, Random' refers to a random-effects meta-analysis with weights based on inverse variances 


\section{C}

Study

Asemi et al (2013) [40]

Asemi et al (2014) [59]

Asemi et al (2016) [20]

Bayat et al (2016) [21]

Ebrahimi et al (2017) [41]

Ejtahed et al (2011) [22]

Feizollahzadeh et al (2017) [36]

Firouzi et al (2017) [49]

Gonai et al (2017) [38]

Hove et al (2015) [23]

Madempudi et al (2019) [47]

Mafi et al (2018) [44]

Mazloom et al (2013) [24]

Mobini et al (2017) [55]

Mohamadshahi et al (2014) [32]

Moroti et al (2012) [37]

Ostadrahimi et al (2015) [33]

Pedersen et al (2016) [50]

Raygan et al (2018) [30]

Razmpoosh et al (2019) [45]

Roshanravan et al (2017) [56]

Sabico et al (2017) [51]

Sabico et al (2019) [52]

Sato et al (2017) [34]

Shakeri et al (2014) [60]

Tajabadi-Ebrahimi et al (2017) [42]

Tonucci et al (2017) [35]

Xu et al (2015) [39]

Zare Javid et al (2020) [29]

\begin{tabular}{|c|c|c|c|c|c|c|}
\hline \multicolumn{3}{|c|}{ Experimental } & \multicolumn{4}{|c|}{ Control } \\
\hline & Mean & SD & $n$ & Mean & SD & $n$ \\
\hline & 1.21 & 0.3 & 27 & 1.16 & 0.2 & 27 \\
\hline & 1.21 & 0.3 & 31 & 1.25 & 0.3 & 31 \\
\hline & 1.12 & 0.3 & 50 & 1.11 & 0.2 & 49 \\
\hline & 1.33 & 0.3 & 20 & 1.04 & 0.3 & 20 \\
\hline & 1.21 & 0.3 & 35 & 1.18 & 0.3 & 35 \\
\hline & 1.24 & 0.3 & 30 & 1.24 & 0.3 & 30 \\
\hline & 1.31 & 1.2 & 20 & 1.27 & 1.2 & 20 \\
\hline & 1.31 & 0.3 & 48 & 1.27 & 0.3 & 53 \\
\hline & 1.39 & 0.4 & 28 & 1.34 & 0.4 & 27 \\
\hline & 1.11 & 0.3 & 18 & 1.11 & 0.3 & 23 \\
\hline & 1.35 & 0.2 & 40 & 1.26 & 0.2 & 39 \\
\hline & 0.88 & 0.2 & 30 & 0.84 & 0.1 & 30 \\
\hline & 1.11 & 0.2 & 16 & 1.13 & 0.4 & 18 \\
\hline & 1.21 & 0.3 & 14 & 1.21 & 0.3 & 15 \\
\hline & 1.31 & 0.2 & 22 & 1.17 & 0.2 & 22 \\
\hline & 1.52 & 0.3 & 10 & 1.43 & 0.4 & 10 \\
\hline & 1.14 & 0.3 & 30 & 1.13 & 0.3 & 30 \\
\hline & 1.09 & 0.3 & 14 & 1.09 & 0.3 & 15 \\
\hline & 1.12 & 0.2 & 30 & 1.11 & 0.2 & 30 \\
\hline & 1.21 & 0.3 & 30 & 1.15 & 0.2 & 30 \\
\hline & 1.18 & 0.3 & 14 & 1.03 & 0.2 & 15 \\
\hline & 1.11 & 0.3 & 39 & 1.01 & 0.3 & 39 \\
\hline & 1.31 & 0.4 & 31 & 0.98 & 0.4 & 30 \\
\hline & 1.41 & 0.4 & 34 & 1.46 & 0.4 & 34 \\
\hline & 1.08 & 0.2 & 26 & 1.11 & 0.2 & 26 \\
\hline [42] & 1.24 & 0.2 & 30 & 1.16 & 0.1 & 30 \\
\hline & 1.53 & 0.3 & 23 & 1.53 & 0.3 & 22 \\
\hline & 1.31 & 0.2 & 44 & 1.45 & 0.3 & 41 \\
\hline & 1.45 & 0.2 & 22 & 1.33 & 0.2 & 22 \\
\hline
\end{tabular}

Random effects model ${ }^{2}=0.0015, \alpha^{2}=36.87(p=06$

806
$(p=0.12)$

813

Heterogeneity: $I^{2}=24 \%, \tau^{2}=0.0015, \chi_{28}^{2}$
Test for overall effect: $z=2.90(p<0.01)$

d

Study

Asemi et al (2013) [40]

Asemi et al (2014) [59]

Asemi et al (2016) [20]

Bayat et al (2016) [21]

Ebrahimi et al (2017) [41]

Ejtahed et al (2011) [22]

Feizollahzadeh et al (2017) [36]

Firouzi et al (2017) [49]

Gonai et al (2017) [38]

Hove et al (2015) [23]

Madempudi et al (2019) [47]

Mafi et al (2018) [44]

Mazloom et al (2013) [24]

Mobini et al (2017) [55]

Mohamadshahi et al (2014) [32]

Moroti et al (2012) [37]

Ostadrahimi et al (2015) [33]

Pedersen et al (2016) [50]

Raygan et al (2018) [30]

Razmpoosh et al (2019) [45]

Roshanravan et al (2017) [56]

Sabico et al (2017) [51]

Sabico et al (2019) [52]

Sato et al (2017) [34]

Shakeri et al (2014) [60]

Tajabadi-Ebrahimi et al (2017) [42]

Tonucci et al (2017) [35]

Xu et al (2015) [39]

Zare Javid et al (2020) [29]

\begin{tabular}{cccccc}
$\begin{array}{c}\text { Experimental } \\
\text { Mean }\end{array}$ & \multicolumn{4}{c}{ SD } & \multicolumn{3}{c}{ Control } & \multicolumn{2}{c}{ Mean } & SD & $n$ \\
& & & & & \\
1.81 & 0.8 & 27 & 1.71 & 0.7 & 27 \\
2.07 & 1.1 & 31 & 2.15 & 1.3 & 31 \\
1.71 & 0.9 & 50 & 1.86 & 0.9 & 49 \\
1.44 & 0.4 & 20 & 2.34 & 1.0 & 20 \\
1.47 & 0.7 & 35 & 1.43 & 0.7 & 35 \\
1.71 & 0.9 & 30 & 1.61 & 0.6 & 30 \\
3.23 & 1.5 & 20 & 3.05 & 1.6 & 20 \\
1.25 & 0.4 & 48 & 1.24 & 0.4 & 53 \\
2.52 & 3.5 & 28 & 1.56 & 0.9 & 27 \\
1.41 & 1.0 & 18 & 1.81 & 1.1 & 23 \\
1.71 & 0.7 & 40 & 1.92 & 1.0 & 39 \\
1.87 & 0.8 & 30 & 2.21 & 0.9 & 30 \\
1.95 & 0.9 & 16 & 2.16 & 0.9 & 18 \\
1.51 & 0.7 & 14 & 2.01 & 1.3 & 15 \\
2.26 & 1.1 & 22 & 2.46 & 1.0 & 22 \\
2.25 & 0.6 & 10 & 2.24 & 0.8 & 10 \\
1.92 & 1.3 & 30 & 1.94 & 0.9 & 30 \\
1.02 & 0.5 & 14 & 0.97 & 0.3 & 15 \\
1.55 & 0.7 & 30 & 1.74 & 0.7 & 30 \\
1.53 & 0.7 & 30 & 1.57 & 0.5 & 30 \\
1.84 & 0.5 & 14 & 1.83 & 0.7 & 15 \\
1.71 & 0.7 & 39 & 2.01 & 0.8 & 39 \\
1.31 & 0.6 & 31 & 2.11 & 1.6 & 30 \\
1.21 & 0.5 & 34 & 1.38 & 1.2 & 34 \\
1.57 & 0.7 & 26 & 2.01 & 1.0 & 26 \\
1.74 & 0.7 & 30 & 1.71 & 0.8 & 30 \\
1.68 & 0.6 & 23 & 1.99 & 0.9 & 22 \\
2.02 & 0.8 & 44 & 1.74 & 1.5 & 41 \\
0.88 & 0.6 & 22 & 0.85 & 0.2 & 22
\end{tabular}

Random effects model

806

Heterogeneity: $I^{2}=21 \%, \tau^{2}=0.0108, \chi_{28}^{2}=35.35(p=0.16)$

$813 \quad 100.0$

Test for overall effect: $z=-2.48(p=0.01)$

Fig. 3 (continued)

probiotics, prebiotics or synbiotics, which is motivated by the possibility of gut microbiota helping individuals with diabetes mellitus achieve favourable metabolic control. To the best of our knowledge, our meta-analysis represents the most comprehensive synthesis to date on the effects of consumption of probiotics, prebiotics and synbiotics on glucose control and 
lipid changes in individuals with diabetes mellitus. Overall, the evidence generated by this review indicates that probiotics, prebiotics and synbiotics do not change LDL-cholesterol levels, they non-significantly decrease $\mathrm{HbA}_{1 \mathrm{c}}$, and they significantly reduce fasting plasma glucose, serum insulin, total cholesterol and triacylglycerol levels and increase HDLcholesterol levels.

Although supplementation reduced fasting plasma glucose, it did not significantly reduce $\mathrm{HbA}_{1 \mathrm{c}}$, which is the standard measure for evaluating long-term glucose control in diabetes. Moreover, the majority of the studies that evaluated insulinaemia as an outcome showed that the interventions resulted in lower insulinaemia, i.e. reduced the severity of insulin resistance. These results were supported by the plasma glucose reduction observed following probiotic, prebiotic or synbiotic supplementation in the majority of studies with reported positive insulin response. Although we have not explored changes in the gut flora in this study, the mechanism of the effect of probiotics on glucose control may be the result of changes in microbiota composition. Several studies in the literature have suggested that consuming probiotics may not lead to a sustainable change in the diversity and the number of bacteria in the gut $[62,63]$; however, even the transition of bacteria through the gut may have some benefits on glycaemic control. This may also explain why long-term changes in $\mathrm{HbA}_{1 \mathrm{c}}$ were not observed in this study. The bacterial strains of L. plantarum, Lactobacillus fermentum, L. casei and L. rhamnosus have shown, in vitro, potent and broadspectrum inhibitory activities on intestinal $\alpha$-glucosidase enzymes as well as the potential to reduce blood glucose in vivo [64]. Thus, supplementation with these strains, observed in at least ten included studies (some studies did not specify the strain of Lactobacillus), could partially explain the results. Furthermore, only eight studies evaluated $\mathrm{HbA}_{1 \mathrm{c}}$ after treatment for more than 12 weeks, but 31 studies evaluated fasting plasma glucose, so it is possible that if all these studies were conducted for more than 12 weeks and evaluated $\mathrm{HbA}_{1 \mathrm{c}}$, a decrease in $\mathrm{HbA}_{1 \mathrm{c}}$ would have been observed.

Low levels of lactate- and butyrate-producing species have previously been associated with adverse impacts on intestinal epithelial barrier function and gut permeability, along with inflammation [65]. However, it is currently unclear whether inflammation can lead to increased intestinal permeability or if it has the opposite effect, since the gut inflammatory responses include an innate immune response mechanism involving Toll-like receptors, producing proinflammatory cytokines and increasing endotoxaemia [66]. A study that evaluated permeability to bacterial products by measuring circulating LPS-binding protein (which facilitates the interaction between LPS and various receptors), intestinal fatty acid binding protein and derived intestinal permeability risk score, reported that all measures were higher in individuals with type 2 diabetes compared with healthy individuals [67]. Moreover, in one study, individuals with type 2 diabetes presented a high rate of gut bacteria in the circulation, providing indirect evidence of bacterial translocation from the gut to the bloodstream [68], which could be related to inflammation and insulin resistance [69]. The inflammatory pathways related to ligands such as bacterial LPS are associated with reduced glucose uptake in insulin-sensitive tissues, increasing insulin requirement [70]. Therefore, probiotic supplementation could be beneficial in reducing inflammation and insulin sensitivity, similar to our results, which showed a reduction in serum insulin levels.

Furthermore, diabetes medication type could be a possible confounder related to the the lack of association for $\mathrm{HbA}_{1 \mathrm{c}}$ because drug-induced modulation of the gut microbiota could be a mechanism by which drugs exert their therapeutic effect in individuals with diabetes, as observed in a cross-sectional study in which individuals with type 2 diabetes using metformin experienced a reduction in the relative abundance of purportedly beneficial mucin-degrading and short-chain fatty acidproducing bacteria [71]. The information about medication type was not clear in most studies analysed and could have interfered with the results of studies evaluating $\mathrm{HbA}_{1 \mathrm{c}}$ as an outcome. In addition, baseline $\mathrm{HbA}_{1 \mathrm{c}}$ level was higher than $8 \%$ in only two studies [39, 58], and it is well known that there is an association between baseline $\mathrm{HbA}_{1 \mathrm{c}}$ and absolute change in $\mathrm{HbA}_{1 \mathrm{c}}$ level in response to glucose-lowering interventions [72].

Despite the small effect sizes, the results highlight an interesting effect of the use of probiotic, prebiotic or synbiotic supplements on lipid profile, which was enhanced (total cholesterol and triacylglycerol levels were decreased, whereas HDL-cholesterol levels were increased). As an enhanced lipid profile is usually associated with a low incidence of diabetesrelated complications, it is tempting to speculate that these results could be reproduced [73]. However, clinical outcomes were not evaluated in the majority of the studies we retrieved, and the follow-up period was too short to determine any longterm effects on morbidity/mortality. Interestingly, a metaanalysis of prospective cohort studies showed that the consumption of fermented milk was associated with a reduced risk of stroke, ischaemic heart disease and cardiovascular mortality events [74]. Therefore, probiotics and synbiotics could be additional treatments for individuals known to be at high risk of cardiovascular events and could be even combined with medications to treat dyslipidaemia.

A recent systematic review and meta-analysis investigated the predictive role of triacylglycerols as a risk factor for cardiovascular disease in people with type 2 diabetes and found that high serum triacylglycerol levels were associated with poor diabetes control and increased risk of cardiovascular disease [75]. Individuals with low levels of HDL-cholesterol have been reported to exhibit a deterioration in beta cell function [76], therefore decreasing triacylglycerol and increasing HDL-cholesterol levels with the use of probiotics, prebiotics or synbiotics may be helpful. 
Our study has some limitations. Data extraction was not blinded, which is a potential source of bias, and the sample sizes of the studies were small. In addition, substantial heterogeneity was identified in the meta-analyses, and to address this, we performed sensitivity analyses to identify the differences between the studies. Moreover, it was a challenge to summarise the results of this review, since different probiotic bacteria were used in the supplements, including several Lactobacillus, Bifidobacterium and Streptococcus strains, some of them together with prebiotics, which may have increased the heterogeneity. Another important factor to consider in the interpretation of our findings is the doses of probiotics, prebiotics and synbiotics, which showed considerable variation among studies, and most studies did not mention the doses used. Another challenge was the wide range of duration of supplementation. Finally, the general quality of the studies led to increased risk of bias in some studies, which may have contributed to the heterogeneity in our analyses.

The strength of this systematic review is that we studied individuals with diabetes and our findings indicate the potential clinical use of probiotics, prebiotics and synbiotics in this group of individuals. Furthermore, we investigated multiple inter-related metabolic outcomes, so that concomitant effects would corroborate the effect of consistent use of probiotics, prebiotics or synbiotics. As we analysed a significant number of studies $(n=38)$, this suggests that the conclusions can be considered reliable.

In conclusion, in individuals with type 2 diabetes mellitus, use of probiotics, prebiotics or synbiotics was associated with improvements in metabolic variables, although the magnitude of these effects was low. Accounting for all included outcomes, our results support the use of probiotics, prebiotics and synbiotics as an adjuvant treatment for metabolic control in type 2 diabetes. The best bacterial strain and concentration remains to be determined. This review highlights the need for further intervention studies to determine the importance of specific bacterial strains, doses and treatment durations.

Acknowledgements The authors would like to express their appreciation to J. F. Schaan (Brazil), who created the art in the graphical abstract. We would like to thank Editage (www.editage.com) for editing and reviewing this manuscript for English language.

Data availability Data are available on request from the authors.

Funding This research received no specific grant from any funding agency in the public, commercial, or not-for-profit sectors.

Authors' relationships and activities The authors declare that there are no relationships or activities that might bias, or be perceived to bias, their work.

Contribution statement BS had full access to all of the data in the study, supervised study and takes responsibility for the integrity of the data and the accuracy of the data analysis. PMB, GHT, AFM and BS designed study. PMB, RR, MS and GL acquired data. PMB analysed data. PMB, GHT, AFM and BS interpreted data. PMB drafted the manuscript. PMB, GHT, RR, MS, GL, AFM and BS revised the manuscript for important intellectual content and approved the version to be published.

\section{References}

1. Zheng Y, Ley SH, Hu FB (2018) Global aetiology and epidemiology of type 2 diabetes mellitus and its complications. Nat Rev Endocrinol 14(2):88-98. https://doi.org/10.1038/nrendo.2017.151

2. Seganfredo FB, Blume CA, Moehlecke M et al (2017) Weight-loss interventions and gut microbiota changes in overweight and obese patients: a systematic review. Obes Rev 18(8):832-851. https://doi. org/10.1111/obr.12541

3. Vallianou NG, Stratigou T, Tsagarakis S (2018) Microbiome and diabetes: where are we now? Diabetes Res Clin Pract 146:111-118. https://doi.org/10.1016/j.diabres.2018.10.008

4. Chakaroun RM, Massier L, Kovacs P (2020) Gut microbiome, intestinal permeability, and tissue bacteria in metabolic disease: perpetrators or bystanders? Nutrients 12(4). https://doi.org/10. 3390/nu12041082

5. Moludi J, Maleki V, Jafari-Vayghyan H, Vaghef-Mehrabany E, Alizadeh M (2020) Metabolic endotoxemia and cardiovascular disease: a systematic review about potential roles of prebiotics and probiotics. Clin Exp Pharmacol Physiol 47(6):927-939. https://doi.org/10.1111/1440-1681.13250

6. Hawkesworth S, Moore SE, Fulford AJ et al (2013) Evidence for metabolic endotoxemia in obese and diabetic Gambian women. Nutr Diabetes 3:e83. https://doi.org/10.1038/nutd.2013.24

7. Hill C, Guarner F, Reid G et al (2014) Expert consensus document. The International Scientific Association for Probiotics and Prebiotics consensus statement on the scope and appropriate use of the term probiotic. Nat Rev Gastroenterol Hepatol 11(8):506514. https://doi.org/10.1038/nrgastro.2014.66

8. Gibson GR, Hutkins R, Sanders ME et al (2017) Expert consensus document: the International Scientific Association for Probiotics and Prebiotics (ISAPP) consensus statement on the definition and scope of prebiotics. Nat Rev Gastroenterol Hepatol 14(8):491-502. https://doi.org/10.1038/nrgastro.2017.75

9. Ebrahimzadeh Leylabadlo H, Sanaie S, Sadeghpour Heravi F, Ahmadian Z, Ghotaslou R (2020) From role of gut microbiota to microbial-based therapies in type 2-diabetes. Infect Genet Evol 81: 104268. https://doi.org/10.1016/j.meegid.2020.104268

10. Everard A, Lazarevic V, Derrien M et al (2011) Responses of gut microbiota and glucose and lipid metabolism to prebiotics in genetic obese and diet-induced leptin-resistant mice. Diabetes 60(11): 2775-2786. https://doi.org/10.2337/db11-0227

11. Yadav H, Jain S, Sinha PR (2007) Antidiabetic effect of probiotic dahi containing Lactobacillus acidophilus and Lactobacillus casei in high fructose fed rats. Nutrition 23(1):62-68. https://doi.org/10. 1016/j.nut.2006.09.002

12. Bejar W, Hamden K, Ben Salah R, Chouayekh H (2013) Lactobacillus plantarum TN627 significantly reduces complications of alloxan-induced diabetes in rats. Anaerobe 24:4-11. https://doi.org/10.1016/j.anaerobe.2013.08.006

13. Andersson U, Bränning C, Ahrné S et al (2010) Probiotics lower plasma glucose in the high-fat fed $\mathrm{C} 57 \mathrm{BL} / 6 \mathrm{~J}$ mouse. Benefic Microbes 1(2):189-196. https://doi.org/10.3920/BM2009.0036

14. Yun SI, Park HO, Kang JH (2009) Effect of Lactobacillus gasseri BNR17 on blood glucose levels and body weight in a mouse model of type 2 diabetes. J Appl Microbiol 107(5):1681-1686. https://doi. org/10.1111/j.1365-2672.2009.04350.x 
15. Hsieh FC, Lee CL, Chai CY, Chen WT, Lu YC, Wu CS (2013) Oral administration of Lactobacillus reuteri GMNL-263 improves insulin resistance and ameliorates hepatic steatosis in high fructosefed rats. Nutr Metab (Lond) 10(1):35. https://doi.org/10.1186/17437075-10-35

16. Honda K, Moto M, Uchida N, He F, Hashizume N (2012) Antidiabetic effects of lactic acid bacteria in normal and type 2 diabetic mice. J Clin Biochem Nutr 51(2):96-101. https://doi.org/10.3164/ jcbn.11-07

17. Kim SH, Huh CS, Choi ID et al (2014) The anti-diabetic activity of Bifidobacterium lactis HY8101 in vitro and in vivo. J Appl Microbiol 117(3):834-845. https://doi.org/10.1111/jam.12573

18. Chen P, Zhang Q, Dang H et al (2014) Antidiabetic effect of Lactobacillus casei CCFM0412 on mice with type 2 diabetes induced by a high-fat diet and streptozotocin. Nutrition 30(9): 1061-1068. https://doi.org/10.1016/j.nut.2014.03.022

19. Valladares R, Sankar D, Li N et al (2010) Lactobacillus johnsonii N6.2 mitigates the development of type 1 diabetes in BB-DP rats. PLoS One 5(5):e10507. https://doi.org/10.1371/journal.pone. 0010507

20. Asemi Z, Alizadeh SA, Ahmad K, Goli M, Esmaillzadeh A (2016) Effects of beta-carotene fortified synbiotic food on metabolic control of patients with type 2 diabetes mellitus: a double-blind randomized cross-over controlled clinical trial. Clin Nutr 35(4): 819-825. https://doi.org/10.1016/j.clnu.2015.07.009

21. Bayat A, Azizi-Soleiman F, Heidari-Beni M et al (2016) Effect of Cucurbita ficifolia and probiotic yogurt consumption on blood glucose, lipid profile, and inflammatory marker in type 2 diabetes. Int J Prev Med 7:30. https://doi.org/10.4103/2008-7802.175455

22. Ejtahed HS, Mohtadi-Nia J, Homayouni-Rad A et al (2011) Effect of probiotic yogurt containing Lactobacillus acidophilus and Bifidobacterium lactis on lipid profile in individuals with type 2 diabetes mellitus. J Dairy Sci 94(7):3288-3294. https://doi.org/10. 3168/jds.2010-4128

23. Hove KD, Brøns C, Færch K, Lund SS, Rossing P, Vaag A (2015) Effects of 12 weeks of treatment with fermented milk on blood pressure, glucose metabolism and markers of cardiovascular risk in patients with type 2 diabetes: a randomised double-blind placebo-controlled study. Eur J Endocrinol 172(1):11-20. https://doi. org/10.1530/EJE-14-0554

24. Mazloom Z, Yousefinejad A, Dabbaghmanesh MH (2013) Effect of probiotics on lipid profile, glycemic control, insulin action, oxidative stress, and inflammatory markers in patients with type 2 diabetes: a clinical trial. Iran J Med Sci 38(1):38-43

25. Nikbakht E, Khalesi S, Singh I, Williams LT, West NP, Colson N (2018) Effect of probiotics and synbiotics on blood glucose: a systematic review and meta-analysis of controlled trials. Eur $\mathbf{J}$ Nutr 57(1):95-106. https://doi.org/10.1007/s00394-016-1300-3

26. Moher D, Liberati A, Tetzlaff J, Altman DG, Group P (2009) Preferred reporting items for systematic reviews and meta-analyses: the PRISMA statement. J Clin Epidemiol 62(10):1006-1012. https://doi.org/10.1016/j.jclinepi.2009.06.005

27. Sterne JAC, Savović J, Page MJ et al (2019) RoB 2: a revised tool for assessing risk of bias in randomised trials. BMJ 366:14898. https://doi.org/10.1136/bmj.14898

28. Cochrane handbook for systematic reviews of interventions, version 6.0 (updated July 2019). Higgins JPT, Thomas J, Chandler J et al (eds). Cochrane, 2019. Available from www. training.Cochrane.org/handbook. Accessed 10 March 2020

29. Zare Javid A, Aminzadeh M, Haghighi-Zadeh MH, Jamalvandi M (2020) The effects of synbiotic supplementation on glycemic status, lipid profile, and biomarkers of oxidative stress in type 1 diabetic patients. A placebo-controlled, double-blind, randomized clinical trial. Diabetes Metab Syndr Obes 13:607-617. https://doi.org/10. 2147/DMSO.S238867
30. Raygan F, Ostadmohammadi V, Bahmani F, Asemi Z (2018) The effects of vitamin $\mathrm{D}$ and probiotic co-supplementation on mental health parameters and metabolic status in type 2 diabetic patients with coronary heart disease: a randomized, double-blind, placebocontrolled trial. Prog Neuro-Psychopharmacol Biol Psychiatry 84(Pt A):50-55. https://doi.org/10.1016/j.pnpbp.2018.02.007

31. Ejtahed HS, Mohtadi-Nia J, Homayouni-Rad A, Niafar M, Asghari-Jafarabadi M, Mofid V (2012) Probiotic yogurt improves antioxidant status in type 2 diabetic patients. Nutrition 28(5):539 543. https://doi.org/10.1016/j.nut.2011.08.013

32. Mohamadshahi M, Veissi M, Haidari F, Javid AZ, Mohammadi F, Shirbeigi E (2014) Effects of probiotic yogurt consumption on lipid profile in type 2 diabetic patients: a randomized controlled clinical trial. J Res Med Sci 19(6):531-536

33. Ostadrahimi A, Taghizadeh A, Mobasseri M et al (2015) Effect of probiotic fermented milk (kefir) on glycemic control and lipid profile in type 2 diabetic patients: a randomized double-blind placebo-controlled clinical trial. Iran J Public Health 44(2):228-237

34. Sato J, Kanazawa A, Azuma K et al (2017) Probiotic reduces bacterial translocation in type 2 diabetes mellitus: a randomised controlled study. Sci Rep 7(1):12115. https://doi.org/10.1038/ s41598-017-12535-9

35. Tonucci LB, Olbrich Dos Santos KM, Licursi de Oliveira L, Rocha Ribeiro SM, Duarte Martino HS (2017) Clinical application of probiotics in type 2 diabetes mellitus: a randomized, double-blind, placebo-controlled study. Clin Nutr 36(1):85-92. https://doi.org/10. 1016/j.clnu.2015.11.011

36. Feizollahzadeh S, Ghiasvand R, Rezaei A, Khanahmad H, Sadeghi A, Hariri M (2017) Effect of probiotic soy milk on serum levels of adiponectin, inflammatory mediators, lipid profile, and fasting blood glucose among patients with type II diabetes mellitus. Probiotics Antimicrob Proteins 9(1):41-47. https://doi.org/10. 1007/s12602-016-9233-y

37. Moroti C, Souza Magri LF, de Rezende Costa M, Cavallini DC, Sivieri K (2012) Effect of the consumption of a new symbiotic shake on glycemia and cholesterol levels in elderly people with type 2 diabetes mellitus. Lipids Health Dis 11:29. https://doi.org/10. 1186/1476-511X-11-29

38. Gonai M, Shigehisa A, Kigawa I et al (2017) Galactooligosaccharides ameliorate dysbiotic Bifidobacteriaceae decline in Japanese patients with type 2 diabetes. Benefic Microbes 8(5): 705-716. https://doi.org/10.3920/BM2016.0230

39. Xu J, Lian F, Zhao L et al (2015) Structural modulation of gut microbiota during alleviation of type 2 diabetes with a Chinese herbal formula. ISME J 9(3):552-562. https://doi.org/10.1038/ ismej.2014.177

40. Asemi Z, Zare Z, Shakeri H, Sabihi SS, Esmaillzadeh A (2013) Effect of multispecies probiotic supplements on metabolic profiles, hs-CRP, and oxidative stress in patients with type 2 diabetes. Ann Nutr Metab 63(1-2):1-9. https://doi.org/10.1159/000349922

41. Ebrahimi ZS, Nasli-Esfahani E, Nadjarzade A, Mozaffari-Khosravi H (2017) Effect of symbiotic supplementation on glycemic control, lipid profiles and microalbuminuria in patients with non-obese type 2 diabetes: a randomized, double-blind, clinical trial. J Diabetes Metab Disord 16:23. https://doi.org/10.1186/s40200-017-0304-8

42. Tajabadi-Ebrahimi M, Sharifi N, Farrokhian A et al (2017) A randomized controlled clinical trial investigating the effect of synbiotic administration on markers of insulin metabolism and lipid profiles in overweight type 2 diabetic patients with coronary heart disease. Exp Clin Endocrinol Diabetes 125(1):21-27. https://doi. org/10.1055/s-0042-105441

43. Khalili L, Alipour B, Asghari Jafar-Abadi M et al (2019) The effects of Lactobacillus casei on glycemic response, serum sirtuin1 and fetuin-A levels in patients with type 2 diabetes mellitus: a randomized controlled trial. Iran Biomed J 23(1):68-77 
44. Mafi A, Namazi G, Soleimani A, Bahmani F, Aghadavod E, Asemi Z (2018) Metabolic and genetic response to probiotics supplementation in patients with diabetic nephropathy: a randomized, doubleblind, placebo-controlled trial. Food Funct 9(9):4763-4770. https:// doi.org/10.1039/c8fo00888d

45. Razmpoosh E, Javadi A, Ejtahed HS, Mirmiran P, Javadi M, Yousefinejad A (2019) The effect of probiotic supplementation on glycemic control and lipid profile in patients with type 2 diabetes: a randomized placebo controlled trial. Diabetes Metab Syndr 13(1):175-182. https://doi.org/10.1016/j.dsx.2018.08.008

46. Sanborn VE, Azcarate-Peril MA, Gunstad J (2020) Lactobacillus rhamnosus $\mathrm{GG}$ and $\mathrm{HbA}_{1 \mathrm{c}}$ in middle age and older adults without type 2 diabetes mellitus: a preliminary randomized study. Diabetes Metab Syndr 14(5):907-909. https://doi.org/10.1016/j.dsx.2020. 05.034

47. Madempudi RS, Ahire JJ, Neelamraju J, Tripathi A, Nanal S (2019) Efficacy of UB0316, a multi-strain probiotic formulation in patients with type 2 diabetes mellitus: a double blind, randomized, placebo controlled study. PLoS One 14(11):e0225168. https://doi.org/10. 1371/journal.pone. 0225168

48. Mirmiranpour H, Huseini HF, Derakhshanian H, Khodaii Z, Tavakoli-Far B (2019) Effects of probiotic, cinnamon, and synbiotic supplementation on glycemic control and antioxidant status in people with type 2 diabetes; a randomized, double-blind, placebo-controlled study. J Diabetes Metab Disord 19(1):53-60. https://doi.org/10.1007/s40200-019-00474-3

49. Firouzi S, Majid HA, Ismail A, Kamaruddin NA, Barakatun-Nisak MY (2017) Effect of multi-strain probiotics (multi-strain microbial cell preparation) on glycemic control and other diabetes-related outcomes in people with type 2 diabetes: a randomized controlled trial. Eur J Nutr 56(4):1535-1550. https://doi.org/10.1007/s00394016-1199-8

50. Pedersen C, Gallagher E, Horton F et al (2016) Host-microbiome interactions in human type 2 diabetes following prebiotic fibre (galacto-oligosaccharide) intake. Br J Nutr 116(11):1869-1877. https://doi.org/10.1017/S0007114516004086

51. Sabico S, Al-Mashharawi A, Al-Daghri NM et al (2017) Effects of a multi-strain probiotic supplement for 12 weeks in circulating endotoxin levels and cardiometabolic profiles of medication naïve T2DM patients: a randomized clinical trial. J Transl Med 15(1):249. https://doi.org/10.1186/s12967-017-1354-X

52. Sabico S, Al-Mashharawi A, Al-Daghri NM et al (2019) Effects of a 6-month multi-strain probiotics supplementation in endotoxemic, inflammatory and cardiometabolic status of T2DM patients: a randomized, double-blind, placebo-controlled trial. Clin Nutr 38(4):1561-1569. https://doi.org/10.1016/j.clnu.2018.08.009

53. Kobyliak N, Falalyeyeva T, Mykhalchyshyn G, Kyriienko D, Komissarenko I (2018) Effect of alive probiotic on insulin resistance in type 2 diabetes patients: randomized clinical trial. Diabetes Metab Syndr 12(5):617-624. https://doi.org/10.1016/j.dsx.2018. 04.015

54. Horvath A, Leber B, Feldbacher N et al (2019) Effects of a multispecies synbiotic on glucose metabolism, lipid marker, gut microbiome composition, gut permeability, and quality of life in diabesity: a randomized, double-blind, placebo-controlled pilot study. Eur J Nutr. https://doi.org/10.1007/s00394-019-02135-w

55. Mobini R, Tremaroli V, Ståhlman M et al (2017) Metabolic effects of Lactobacillus reuteri DSM 17938 in people with type 2 diabetes: a randomized controlled trial. Diabetes Obes Metab 19(4):579-589. https://doi.org/10.1111/dom.12861

56. Roshanravan N, Mahdavi R, Alizadeh E et al (2017) Effect of butyrate and inulin supplementation on glycemic status, lipid profile and glucagon-like peptide 1 level in patients with type 2 diabetes: a randomized double-blind, placebo-controlled trial. Horm Metab Res 49(11):886-891. https://doi.org/10.1055/s-0043119089
57. Sheth M, Chand V, Thakuria (2015) Inflated levels of SCFA, Bifidobacteria and Lactobacillus improves the status of pre hypertension and type 2 diabetes mellitus in subjects residing in north east India - a randomized control trial with synbiotic supplementation. Int J Curr Pharm Res 7(3):33-36

58. Gargari BP, Dehghan P, Aliasgharzadeh A, Asghari Jafar-Abadi M (2013) Effects of high performance inulin supplementation on glycemic control and antioxidant status in women with type 2 diabetes. Diabetes Metab J 37(2):140-148. https://doi.org/10. 4093/dmj.2013.37.2.140

59. Asemi Z, Khorrami-Rad A, Alizadeh SA, Shakeri H, Esmaillzadeh A (2014) Effects of synbiotic food consumption on metabolic status of diabetic patients: a double-blind randomized cross-over controlled clinical trial. Clin Nutr 33(2):198-203. https://doi.org/ 10.1016/j.clnu.2013.05.015

60. Shakeri H, Hadaegh H, Abedi F et al (2014) Consumption of synbiotic bread decreases triacylglycerol and VLDL levels while increasing HDL levels in serum from patients with type-2 diabetes. Lipids 49(7):695-701. https://doi.org/10.1007/s11745-014-3901-z

61. Tajadadi-Ebrahimi M, Bahmani F, Shakeri H et al (2014) Effects of daily consumption of synbiotic bread on insulin metabolism and serum high-sensitivity C-reactive protein among diabetic patients: a double-blind, randomized, controlled clinical trial. Ann Nutr Metab 65(1):34-41. https://doi.org/10.1159/000365153

62. Zmora N, Zilberman-Schapira G, Suez J et al (2018) Personalized gut mucosal colonization resistance to empiric probiotics is associated with unique host and microbiome features. Cell 174(6):1388 1405.e21. https://doi.org/10.1016/j.cell.2018.08.041

63. Gargari G, Taverniti V, Koirala R, Gardana C, Guglielmetti S (2020) Impact of a multistrain probiotic formulation with high bifidobacterial content on the fecal bacterial community and short-chain fatty acid levels of healthy adults. Microorganisms 8(4):492. https://doi.org/10.3390/microorganisms8040492

64. Panwar H, Calderwood D, Grant IR, Grover S, Green BD (2014) Lactobacillus strains isolated from infant faeces possess potent inhibitory activity against intestinal alpha- and beta-glucosidases suggesting anti-diabetic potential. Eur J Nutr 53(7):1465-1474. https://doi.org/10.1007/s00394-013-0649-9

65. de Goffau MC, Luopajärvi K, Knip M et al (2013) Fecal microbiota composition differs between children with $\beta$-cell autoimmunity and those without. Diabetes 62(4):1238-1244. https://doi.org/10.2337/ db12-0526

66. Sharma S, Tripathi P (2019) Gut microbiome and type 2 diabetes: where we are and where to go? J Nutr Biochem 63:101-108. https:// doi.org/10.1016/j.jnutbio.2018.10.003

67. Cox AJ, Zhang P, Bowden DW et al (2017) Increased intestinal permeability as a risk factor for type 2 diabetes. Diabetes Metab 43(2):163-166. https://doi.org/10.1016/j.diabet.2016.09.004

68. Sato J, Kanazawa A, Ikeda F et al (2014) Gut dysbiosis and detection of "live gut bacteria" in blood of Japanese patients with type 2 diabetes. Diabetes Care 37(8):2343-2350. https://doi.org/10.2337/ dc13-2817

69. Li X, Watanabe K, Kimura I (2017) Gut microbiota dysbiosis drives and implies novel therapeutic strategies for diabetes mellitus and related metabolic diseases. Front Immunol 8:1882. https://doi. org/10.3389/fimmu.2017.01882

70. Ferrari F, Bock PM, Motta MT, Helal L (2019) Biochemical and molecular mechanisms of glucose uptake stimulated by physical exercise in insulin resistance state: role of inflammation. Arq Bras Cardiol 113(6):1139-1148. https://doi.org/10.5935/abc.20190224

71. de la Cuesta-Zuluaga J, Mueller NT, Corrales-Agudelo V et al (2017) Metformin is associated with higher relative abundance of mucin-degrading Akkermansia muciniphila and several short-chain fatty acid-producing microbiota in the gut. Diabetes Care 40(1):54 62. https://doi.org/10.2337/dc16-1324 
72. DeFronzo RA, Stonehouse AH, Han J, Wintle ME (2010) Relationship of baseline $\mathrm{HbA}_{1 \mathrm{c}}$ and efficacy of current glucoselowering therapies: a meta-analysis of randomized clinical trials. Diabet Med 27(3):309-317. https://doi.org/10.1111/j.1464-5491. 2010.02941.x

73. Keng MJ, Tsiachristas A, Leal J, Gray A, Mihaylova B (2019) Impact of achieving primary care targets in type 2 diabetes on health outcomes and healthcare costs. Diabetes Obes Metab 21(11):2405-2412. https://doi.org/10.1111/dom.13821

74. Companys J, Pla-Pagà L, Calderón-Pérez L et al (2020) Fermented dairy products, probiotic supplementation, and cardiometabolic diseases: A systematic review and meta-analysis. Adv Nutr 11(4): 834-863. https://doi.org/10.1093/advances/nmaa030
75. Ye X, Kong W, Zafar MI, Chen LL (2019) Serum triglycerides as a risk factor for cardiovascular diseases in type 2 diabetes mellitus: a systematic review and meta-analysis of prospective studies. Cardiovasc Diabetol 18(1):48. https://doi.org/10.1186/s12933019-0851-z

76. Fiorentino TV, Succurro E, Marini MA et al (2020) HDL cholesterol is an independent predictor of $\beta$ cell function decline and incident type 2 diabetes: a longitudinal study. Diabetes Metab Res Rev. https://doi.org/10.1002/dmrr.3289

Publisher's note Springer Nature remains neutral with regard to jurisdictional claims in published maps and institutional affiliations. 